In Crescendo. Institucional. 2015; 6(2): 104-113

Fecha de recepción: 25 de octubre de 2015

Fecha de aceptación: 10 de noviembre de 2015

\title{
NIVELES DE CONOCIMIENTO SOBRE NEUROCIENCIA Y SU APLICACIÓN EN LOS PROCESOS EDUCATIVOS
}

\section{LEVELS OF KNOWLEDGE OF NEUROSCIENCE AND ITS APPLICATION IN EDUCATIONAL PROCESS}

\author{
Mario Enrique Nizama Reyes*
}

\begin{abstract}
RESUMEN
T a presente investigación tuvo como objetivo determinar los niveles de conocimiento

Ly aplicación de la neurociencia en los procesos educativos entre los participantes de maestrías en Ciencias de la Educación de la Región Piura, durante el semestre 20152. La investigación fue descriptivo-cuantitativa, siendo la población igual a la suma de tres grupos de maestristas en Ciencias de la Educación, equivalente a 67 docentes y la muestra se obtuvo de un muestreo no probabilístico, por conveniencia de la investigación, específicamente los maestristas que se desempeñan como docentes de aula en la educación superior; la cual resultó igual a 32 docentes a quienes se les aplicó, mediante la técnica de encuesta, dos cuestionarios: uno de recojo de los niveles de conocimiento sobre neurociencia y el otro de recojo de niveles de aplicación de la neurociencia en los procesos educativos. Los resultados que se obtuvieron fueron los siguientes: en cuanto a los niveles de conocimiento sobre neurociencia, se registró un limitado porcentaje de conocimientos, pues en promedio solo el 9 \% alcanzó el nivel alto; mientras que para los niveles de aplicación de la neurociencia en los procesos educativos, sí se obtuvo un significativo porcentaje de aplicación ya que en promedio se registró $20 \%$ en el nivel alto. En cuanto a las conclusiones, se puede indicar en primer lugar que los niveles de conocimiento y aplicación de la neurociencia en los procesos educativos, entre los participantes de la presente investigación, son predominantemente bajos e intermedios. En segundo lugar, que los conocimientos más destacados son el funcionamiento neuronal y la relación entre cerebro y aprendizaje. Para concluir, podemos indicar que las aplicaciones en los procesos
\end{abstract}

* Magíster en Ciencias, mención en Investigación y Docencia.Docente tutor de la Universidad Católica Los Ángeles de Chimbote, filial Piura. 
educativos con mejores niveles son el trabajo en modalidad paralela y comprensión a través de la vivencia.

Palabras clave: neurociencia, neuroeducación, procesos cognitivos.

\begin{abstract}
This research aimed to determine the levels of knowledge and application of neuroscience in education among the participants of master Science in Education Piura region, during the semester 2015-2. The research was descriptive - quantitative, with the population equal to the sum of three groups master students in Educational Sciences, equivalent to 67 teachers and the sample was obtained from a non-probability sampling, for convenience of investigation, specifically master students that serve as classroom teachers in higher education which was equal to 32 teachers who were applied through technical survey, two questionnaires, one pick-up levels of knowledge about neuroscience and the other levels pickup application of neuroscience in education. The results obtained were as follows: in terms of levels of knowledge about neuroscience a limited percentage of recorded knowledge, because on average only $9 \%$ reached the highest level; whereas for levels of application of neuroscience in education if a significant percentage of application was obtained as an average of $20 \%$ was recorded in the high level. As for the findings it may indicate, first, that the levels of knowledge and application of neuroscience in education among the participants of this research are predominantly low and intermediate. Second, that knowledge is the most prominent neuronal function and the relationship between brain and learning. To conclude, indicate that applications in education with higher levels are working in parallel mode and understanding through experience.
\end{abstract}

Keywords: neuroscience, neuroeducation, cognitive processes.

\title{
INTRODUCCIÓN
}

A pesar de tener casi 100 años de existencia como la ciencia del abordaje y estudio interdisciplinario del cerebro, la neurociencia es una ciencia incipiente y prolífica en nuestro país y augura una verdadera revolución o cambio de paradigma en la manera de entender al ser humano como una unidad indisociable: biológica, psicológica y social. Nuevas tecnologías, como la resonancia magnética funcional, permiten actualmente ver al cerebro funcionando en vivo, posibilitando una mayor y mejor comprensión del sustrato anatómico-cerebral que subyace a la cognición y compleja conducta humana.

El cerebro se encuentra dividido en dos grandes estructuras o hemisferios con características funcionales singulares pero complementarias. Estos dos hemisferios, izquierdo y derecho respectivamente, se encuentran interconectados por un grueso haz 
de fibras nerviosas, alrededor de doscientos millones, que le permite interactuar con el mundo en forma unificada, como un todo. No obstante, cada hemisferio cerebral posee ciertas particularidades que lo hacen único, tal es así que el hemisferio cerebral izquierdo trabaja con una modalidad secuencial, procesa predominantemente información simbólica no analógica, el lenguaje verbal y significados semánticos verbales; es analítico, cuantitativo y matemático, también trabaja los procesos cognitivos conscientes como la percepción, atención y memoria, la afectividad social aprendida y también es responsable de las construcciones sociales. Mientras que el hemisferio cerebral derecho presenta características complementarias no menos importantes como son trabajar con una modalidad simultánea o paralela, es holístico y global; sigue una lógica analógica, no verbal; permite la comprensión de los hechos a través de la vivencia; trabaja los procesos cognitivos no conscientes como la atención y memoria no consciente, la afectividad primaria y es responsable de los procesos creativos y el arte en general (Blakemore, 2007).

Las sinapsis son uniones especializadas mediante las cuales las células del sistema nervioso envían señales de unas a otras y a células no neuronales. En la infancia el cerebro forma sinapsis en mayor cantidad que en la edad adulta. El número de sinapsis por unidad volumen de tejido (la densidad sináptica) en la capa cortical exterior del cerebro cambia a lo largo de la vida en monos y humanos. Los humanos recién nacidos tienen menor densidad sináptica que los adultos. Sin embargo, durante los meses que siguen al nacimiento, el cerebro del bebé comienza a formar sinapsis en exceso respecto de los niveles del adulto. En humanos, a los 4 años de edad, las densidades sinápticas llegan a su pico en todas las áreas cerebrales, al menos $50 \%$ por encima de los niveles adultos. Durante la infancia, las densidades sinápticas se mantienen por encima de las del adulto, y hacia la pubertad, un proceso de eliminación sináptica ocurre, reduciendo los niveles.

Ahora cabe cuestionarse por qué un educador debe tener conocimientos acerca del cerebro. Pues porque al conocer algunas de las particularidades del sistema nervioso y del cerebro, entenderemos cómo el ser humano se desarrolla y cómo el aprendizaje influye en este proceso. Al comprender cómo aprende el cerebro, automáticamente repensaremos el papel que desempeñamos como agentes educativos y buscaremos mejorar la calidad de nuestro trabajo y de nuestros programas, la metodología que usamos y la calidad de las actividades que proponemos. Este conocimiento nos llevará a actuar con mayor efectividad y creatividad, en pro de lo que realmente debemos hacer: propiciar el desarrollo del individuo y de su potencial humano.

Por todo lo antes expuesto y detallado se justifica el presente trabajo de investigación, pues a la luz de los nuevos descubrimientos de la neurociencia, no es posible ignorar los enormes beneficios que esta ciencia puede representar para los aprendizajes de los estudiantes. Por lo tanto, se plantea el siguiente problema: ¿Cuáles son los niveles de conocimiento y aplicación de la neurociencia en los procesos educativos entre los participantes de maestrías en Ciencias de la Educación de la Región Piura, durante el semestre 2015-2? Para dar respuesta a este problema, se planteó el siguiente objetivo general: Determinar los niveles de conocimiento y aplicación de la neurociencia en los 
procesos educativos entre los participantes de maestrías en Ciencias de la Educación de la Región Piura, durante el semestre 2015-2.

\section{MATERIAL Y MÉTODOS}

En la investigación la población estuvo constituida por la suma de tres grupos de maestristas en Ciencias de la Educación: 28 maestristas de la Universidad César Vallejo Piura (UCV), 21 maestristas de la Universidad Nacional de Piura (UNP) y 18 maestristas de la Universidad de Piura (UDEP). Es decir la población fue de 67 maestristas. Para determinar la muestra, el criterio de selección es un muestreo no probabilístico, por conveniencia de la investigación, específicamente los maestristas que se desempeñan como docentes de aula en la educación superior (técnica y universitaria): 12 maestristas de la Universidad César Vallejo - Piura (UCV), 10 maestristas de la Universidad Nacional de Piura (UNP) y 10 maestristas de la Universidad de Piura (UDEP). Por tanto, la muestra fue de 32 maestristas.

Se utilizó la técnica de la encuesta y los instrumentos específicos fueron dos cuestionarios: uno de recojo de los niveles de conocimiento sobre neurociencia (con 6 ítems) y el otro de recojo de niveles de aplicación de la neurociencia en los procesos educativos (con 6 ítems). Para cada uno de los ítems de los dos cuestionarios, se incluyó una caja de texto para que los docentes justificaran brevemente su evaluación (no mayor a dos líneas de texto en promedio) a fin de hacer un mejor análisis de los resultados (Sandin, 2003).

En el proceso de la investigación se utilizó el método descriptivo-cuantitativo. Es descriptivo, por cuanto se obtuvieron datos y se hicieron mediciones de cada uno de los ítems de los niveles de conocimiento sobre neurociencia y de los niveles de aplicación de la neurociencia en los procesos educativos; y es cuantitativo, por cuanto se han recogido y analizado datos definidos, exactos y precisos de cada uno de esos ítems de las dos dimensiones de evaluación utilizadas.

\section{RESULTADOS}

La escala de medición que se utilizó fue una escala de Likert modificada, la cual se empleó para la interpretación de los resultados según el siguiente detalle:

1 = Muy bajo (no se tienen ni conceptos ni referencias específicas)

2 = Bajo (solo se tienen referencias definidas, mas no conceptos especificos)

$3=$ Intermedio (se tienen conceptos especificos, pero no significativos)

$4=$ Alto (se tienen conceptos significativos y generalizables)

$5=$ Muy alto (se tienen conceptos prácticos, aplicables e innovadores) 
En los resultados de los niveles de conocimiento sobre neurociencia, de acuerdo a la Tabla 1 (de la sección anexos) podemos destacar que, de los seis ítems estudiados, ninguno cae en los valores extremos de la escala utilizada, es decir no hay valores para el nivel de respuesta 1 (nivel muy bajo), ni para el nivel de respuesta 5 (nivel muy alto).

Respecto al ítem del significado de neurociencias, se registra su mayor porcentaje en el nivel de respuesta 2 (nivel bajo), siendo su mínimo porcentaje el nivel 4 (nivel alto) con $6 \%$.

Para el ítem acerca del conocimiento del sistema nervioso, su mayor porcentaje también lo tiene el nivel de respuesta 2 (nivel bajo) y el porcentaje mínimo es para el nivel de respuesta 3 (intermedio); no se presentan valores en los niveles 1, 4 y 5 (niveles muy bajo, alto y muy alto, respectivamente).

Con respecto a las características y áreas del cerebro también en nivel de respuesta 2 (nivel bajo), presenta el mayor porcentaje, aunque hay un $9 \%$ de participantes que muestra un nivel de respuesta 4 (nivel alto). Para el ítem de conocimiento del funcionamiento neuronal, los valores suben hacia los niveles positivos, así tenemos un $66 \%$ de respuesta para el nivel de respuesta 3 (nivel intermedio) y un $16 \%$ para el nivel de respuesta 4 (nivel alto).

Para el significado de plasticidad cerebral tanto en el nivel de respuesta 2 (nivel bajo) como 3 (nivel intermedio), se alcanzó un 47 \% para cada nivel, aunque también figura un $6 \%$ para el nivel de respuesta 4 (nivel alto). Y para el nivel de conocimiento de la relación entre cerebro y aprendizaje, se alcanzó un $18 \%$ para el nivel de respuesta 4 (nivel alto); se convierte así en el mejor porcentaje de este nivel en la Tabla 1, además de un 66 \% para el nivel intermedio y solo $16 \%$ para el nivel bajo.

Respecto a los resultados de los niveles de aplicación de la neurociencia en los procesos educativos, tal como se observa en la Tabla 2 y al igual que en la Tabla 1, no se registró en los seis ítems estudiados ningún valor para el nivel de respuesta 1 (nivel muy bajo), ni para el nivel de respuesta 5 (nivel muy alto).

Para el caso del trabajo en modalidad paralela, tanto el nivel de respuesta 4 (nivel alto) como el nivel de respuesta 2 (nivel bajo) alcanzaron un $28 \%$, mientras que para el trabajo holístico y global hay un $37 \%$ para el nivel de respuesta 3 (nivel intermedio) y un considerable $22 \%$ para el nivel de respuesta 4 (nivel alto).

En lo que respecta al uso de la lógica analógica, se presentó un valor del 63 \% para el nivel bajo de aplicación y solo un 6 \% para el nivel alto de aplicación; no sucedió lo mismo para la comprensión a través de la vivencia, donde el nivel alto de aplicación llegó a un nada despreciable $28 \%$ frente a un $13 \%$ del nivel bajo de aplicación.

Para la edificación de la afectividad primaria, la predominancia la tiene el nivel intermedio de aplicación, con un $63 \%$ y solo un $15 \%$ para los niveles altos de aplicación de este ítem tan trascendental. 
Finalmente, para el ítem referido al empleo de procesos creativos se obtuvo un $28 \%$ para el nivel bajo de aplicación, un $47 \%$ para el nivel intermedio y un $25 \%$ para el nivel alto de aplicación.

\section{DISCUSIÓN}

Al analizar los resultados de los niveles tanto del conocimiento como de las aplicaciones de la neurociencia en los procesos educativos reflejados en la Tabla 1 y Tabla 2 respectivamente, se puede rápidamente apreciar el poco significado e impacto que ha tenido esta trascendental ciencia entre los docentes participantes de la presente investigación.

Empezando por la evaluación de los niveles de conocimiento sobre neurociencia reflejada en la Tabla 1, específicamente sobre el conocimiento de lo más básico que es el significado o definición de neurociencia, solo un $6 \%$ está en un nivel alto, es decir tiene un concepto significativo y generalizable de que la neurociencia estudia el cerebro y el sistema nervioso desde un enfoque multidisciplinario (biológico, químico, físico, electrofisiológico, informático, farmacológico, genético, entre otros) mientras que la gran mayoría (60\% de los participantes) solo tiene referencias pero no conceptos específicos de neurociencia, con lo cual se puede deducir que no existe una verdadera base conceptual para su correcta aplicación (Monereo, 2001).

Los niveles de conocimiento sobre el sistema nervioso son aún más bajos, pues un $84 \%$ de los participantes se ubica precisamente en el nivel 2 (nivel bajo), lo cual indica que no hay conceptos específicos del sistema nervioso central (compuesto por cerebro y médula espinal) y menos aún del sistema nervioso periférico, lo que nos lleva a inferir que no hay una comprensión de cómo el sistema nervioso central controla todos los procesos que ocurren en nuestro cuerpo, recibiendo información de los diferentes órganos y enviando instrucciones para que todo funcione correctamente, ni tampoco que está compuesto por dos tipos de células: la neurona (unidad básica estructural del sistema nervioso) y la neuroglia (especie de sostén en la estructura de comunicación de las células nerviosas); ni de lo que es sinapsis (transmisión de impulsos nerviosos) y la neurotransmisión (a través de los mensajeros químicos llamados neurotransmisores).

Para el caso del ítem 3, hay un significativo incremento en los niveles de conocimiento intermedio (31 \%) y alto (9 \%), lo cual nos indica que hay conceptos específicos y hasta generalizables con respecto a que el cerebro está compuesto por tres grandes partes (el cerebro anterior o telencéfalo, el cerebro medio o mesencéfalo, y el cerebro posterior), además que posee dos hemisferios (el derecho e izquierdo), conectados por la parte central mediante un haz de fibras nerviosas (el cuerpo calloso), que les permite intercambiar información. Cada hemisferio tiene cuatro lóbulos: el frontal (involucrado en la planificación, el razonamiento, el control de la emoción y otras importantes funciones de orden superior), el parietal (encontramos la percepción del gusto, el tacto y la temperatura), el occipital (procesa lo que vemos y, entre otras funciones, está atento 
a la forma, color y movimiento) y el temporal (soporta muchas funciones tales como el procesamiento de volumen y la frecuencia en la música, el sonido y el habla) (Iacoboni, 2009)). En anexos, imagen 1 se esquematizan las partes del cerebro.

Con relación al funcionamiento neuronal, también existen porcentajes significativos para el nivel 4 (nivel alto con $16 \%$ ) y el nivel 3 (nivel intermedio con $66 \%$ ); es decir, existen conocimientos definidos sobre que una neurona consta de un soma, que es donde se encuentra el núcleo; un axón, que es como el tubo conductor que transporta la energía del núcleo a los botones terminales; y las dendritas, las cuales son las ramificaciones contactadas con otras neuronas a través de los botones sinápticos. Todas las funciones que experimentamos se generan por la conexión entre neuronas, a través de un proceso que se conoce como potencial de acción, el cual se da cuando una neurona se activa descargando un impulso (que a través de iones sodio invierte el voltaje a través de la misma neurona), lo que origina la liberación de sustancias químicas llamadas neurotransmisores desde el botón terminal o sináptico de la neurona. Estas sustancias cruzan el espacio sináptico y son aceptadas por receptores de dendritas de otra neurona, y ahí se concluye el potencial de acción que continuamente se activa y repite y produce así la actividad cerebral (Doidge, 2007).

En cuanto al significado plasticidad cerebral, los mayores porcentajes están tanto para el nivel 3 (nivel intermedio con 47 \%) como el nivel 2 (nivel bajo también con 47 \%). Este conocimiento específico se refiere a que la característica más sorprendente del cerebro es su plasticidad, o la capacidad de cambiar su estructura y organización a lo largo de su vida, en respuesta a las influencias genéticas y ambientales. El proceso de aprendizaje permite a la persona su adaptación al entorno cultural. Esto requiere de cambios en el sistema nervioso, de ahí la importancia de la plasticidad del cerebro para poder tener capacidad de aprendizaje y de adaptarse a nuevas situaciones.

Finalmente, en la Tabla 1, para el ítem de relación entre cerebro y aprendizaje, se obtiene el mejor porcentaje del nivel 4 (nivel alto con un 18 \%). Este conocimiento nos indica que el cerebro ha evolucionado para educar y ser educado, a menudo de manera instintiva y sin esfuerzo. El cerebro es la máquina gracias a la cual se producen todas las formas de aprendizaje, de la misma manera es también el mecanismo natural que pone límites en el aprendizaje; determina lo que puede ser aprendido, cuánto y con qué rapidez. Se considera que el cerebro es feliz analizando y comparando situaciones nuevas, por lo que una enseñanza en la que se presenten al cerebro nuevas y variadas alternativas será mucho mejor que aquella en la que el cerebro tenga que dar siempre un mismo tipo de respuesta, aunque esta sea por lo general correcta (Ortiz, 2009).

En la evaluación de los niveles de aplicación de la neurociencia en los procesos educativos reflejada en la Tabla 2, el trabajo de modalidad paralela llega a tener una aplicación del $28 \%$ en el nivel alto (nivel 4). Como ejemplificación, podemos indicar lo siguiente: si un estudiante lee un libro o un manual sobre el antiguo Imperio Romano, se activa el hemisferio cerebral izquierdo gracias a su modalidad lógico-verbal, lo que implica un menor procesamiento de información en una unidad de tiempo, en comparación al 
hemisferio contralateral. Si se provee al alumno de experiencias sensoriales enriquecidas, se favorece la activación del hemisferio cerebral derecho, que posee la ventaja adicional de procesar una mayor cantidad de ítems en forma simultánea a través de múltiples canales sensoriales. En este sentido, la utilización de videos podría representar sin duda una ganancia adicional. En resumen, insume mucho menos tiempo ver una película que leer el guion de la misma.

Para el caso del trabajo holístico y global, un 22 \% está en un nivel de aplicación alto. En esta aplicación de la neurociencia a la educación, se presume un error pedagógico presentar materias o asignaturas de forma aislada o inconexa, o desconectada. Por ejemplo, nuestra Historia Nacional Contemporánea podría ser enseñada asociada o inserta dentro de un marco mayor o global de la Historia Universal. Para continuar con la ejemplificación anterior, la enseñanza de la Historia del Antiguo Imperio Romano se podría emparejar y conectar con asignaturas como Geografía, Filosofía, Educación Cívica, Economía, Arte, etc.

En el ítem uso de lógica analógica, solo un 6 \% está en un nivel de aplicación alto, pues este punto implica la trasmisión de conceptos más o menos complejos o abstractos apelando a la metáfora y a todas las formas de imágenes visuales. Dicho en otras palabras, se sugiere ilustrar lo que se enseña, más allá de las palabras, en un formato acorde o atractivo a los intereses artísticos del estudiante. Por ejemplo, se podrían desarrollar cómics o historietas sobre el Imperio Romano, o crear videos multisensoriales, videojuegos interactivos, juegos de roles, etc.

La aplicación de la comprensión a través de la vivencia es una de la mayores, pues llegó a un $28 \%$ en el nivel alto, y definitivamente es una de las aplicaciones que más se debe explotar, pues el cerebro humano _en tanto órgano plástico, permeable_, experimenta cambios físicos reales de manera constante y como consecuencia de una permanente interacción con el medio ambiente. Las experiencias de vida esculpen literalmente el cerebro, propiciando el desarrollo y crecimiento del árbol dendrítico entre las neuronas, que son las células cerebrales implicadas en todos los procesos cognitivos y pilares y sustrato biológico del aprendizaje; por ende, se fortalece la inteligencia práctica.

En cuanto a la edificación de afectividad primaria, el $15 \%$ alcanzado en el nivel alto refleja una alta consideración del sistema atencional, pilar fundamental sobre el que se apoya todo aprendizaje, pues la atención y emoción son dos aspectos de la cognición en permanente interacción. Dicho de otra manera, el estado de ánimo, el humor y las emociones comandan la atención. Un clima emocional positivo dentro del aula entre compañeros y profesor puede erigirse como uno de los mayores facilitadores para el aprendizaje (Eichenbaum, 2003).

Finalmente, en el ítem empleo de procesos creativos el nivel alto de aplicación llegó hasta un $25 \%$, pues es una aplicación que posibilita crear experiencias de aprendizaje compatibles con la modalidad de funcionamiento del hemisferio cerebral derecho, mediante la simulación o representación actoral de sucesos históricos, el dibujo, la educación plástica, la música e incluso la lengua y las letras, con el desarrollo de novelas 
cuya trama aparezca enmarcada dentro de un contexto sociohistórico específico que se pretende enseñar, o cuentos y fábulas que ilustren o metaforicen contenidos abstractos, etc.

Con base en todos los resultados que se han podido presentar, sistematizar y discutir previamente, como conclusiones del presente trabajo, podemos mencionar las siguientes:

A Losnivelesdeconocimientoyaplicación delaneurocienciaenlosprocesoseducativos entre los participantes de la presente investigación son predominantemente bajos e intermedios, lo que reafirma que la neurociencia en nuestra realidad es incipiente, relegada y hasta olvidada y postergada, pero a la vez prolífica.

A Los conocimientos más destacados, es decir los más significativos y generalizables son el funcionamiento neuronal ( $16 \%$ en el nivel alto) y la relación entre cerebro y aprendizaje (18 \% en el nivel alto), lo cual es indicador que, a pesar de no poseer conocimientos detallados de neurociencia, se entiende su importancia y necesidad.

A Las aplicaciones en los procesos educativos con mejores niveles son el trabajo en modalidad paralela (28 \% en el nivel alto) y comprensión a través de la vivencia (28 \% en el nivel alto), lo cual reafirma que, a pesar de no poseer arraigados conocimientos, existe un esfuerzo docente por aplicar la neurociencia para mejorar la calidad de los procesos de enseñanza y aprendizaje.

\section{REFERENCIAS BIBLIOGRÁFICAS}

Blakemore, S. (2007). Cómo aprende el Cerebro. Barcelona: Editorial Planeta.

Doidge, N. (2007). The Brain that changes itself. Madrid: Editorial Aguilar.

Eichenbaum, H. (2003). Neurociencia cognitiva de la memoria. Barcelona: Editorial Ariel.

Iacoboni, M. (2009). Las neuronas espejo. Buenos Aires: Editorial Katz.

Monereo, C. (2001). La enseñanza estratégica: enseñar para la autonomía. Barcelona: Editorial Graó.

Ortiz, T. (2009). Neurociencia y educación. Madrid: Alianza Editorial.

Sandin, M. (2003). Investigación cualitativa en educación. México: McGraw-Hill. 


\section{ANEXOS}

Tabla 1. Niveles de conocimiento sobre neurociencia

\begin{tabular}{llcccccccccc} 
N & \multicolumn{1}{c}{ Ítem } & 5 & 4 & 3 & 2 & 1 & 5 & 4 & 3 & 2 & 1 \\
\hline 1 & Significado de neurociencias & 0 & 2 & 11 & 19 & 0 & 0 & 6 & 34 & 60 & 0 \\
2 & Sistema Nervioso & 0 & 0 & 5 & 27 & 0 & 0 & 0 & 16 & 84 & 0 \\
3 & Características y áreas del cerebro & 0 & 3 & 10 & 19 & 0 & 0 & 9 & 31 & 60 & 0 \\
4 & Funcionamiento neuronal & 0 & 5 & 21 & 6 & 0 & 0 & 16 & 66 & 18 & 0 \\
5 & Significado de plasticidad cerebral & 0 & 2 & 15 & 15 & 0 & 0 & 6 & 47 & 47 & 0 \\
6 & Relación entre cerebro y aprendizaje & 0 & 6 & 21 & 5 & 0 & 0 & 18 & 66 & 16 & 0 \\
\hline$\quad$ Promedio & & & & & & 0 & 9 & 43 & 48 & 0
\end{tabular}

Tabla 2.Niveles de aplicación de la neurociencia en los procesos educativos

\begin{tabular}{clccccccccccc}
$\mathrm{N}^{\mathbf{0}}$ & \multicolumn{1}{c}{ Ítem } & 5 & 4 & 3 & 2 & 1 & 5 & 4 & 3 & 2 & 1 \\
\hline 1 & Trabajo en modalidad paralela & 0 & 9 & 14 & 9 & 0 & 0 & 28 & 44 & 28 & 0 \\
2 & Trabajo holístico y global & 0 & 7 & 15 & 10 & 0 & 0 & 22 & 47 & 31 & 0 \\
3 & Uso de lógica analógica & 0 & 2 & 10 & 20 & 0 & 0 & 6 & 31 & 63 & 0 \\
4 & Comprensión a través de la vivencia & 0 & 9 & 19 & 4 & 0 & 0 & 28 & 59 & 13 & 0 \\
5 & Edificación de afectividad primaria & 0 & 5 & 20 & 7 & 0 & 0 & 15 & 63 & 22 & 0 \\
6 & Empleo de procesos creativos & 0 & 8 & 15 & 9 & 0 & 0 & 25 & 47 & 28 & 0 \\
\hline \multicolumn{1}{c}{ Promedio } & & & & & & 0 & 20 & 49 & 31 & 0
\end{tabular}

Figura 1. Partes del cerebro

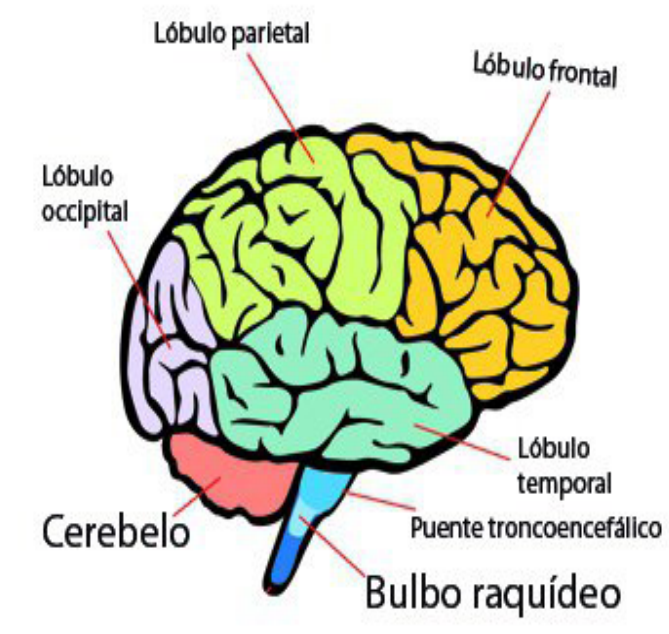

